\title{
Distance of the Posterior Interosseous Nerve from the Radial Head during Elbow Arthroscopy: An Anatomical Study
}

\author{
Paolo Arrigoni ${ }^{1,2, *}$ Davide Cucchi ${ }^{1,3, *}$ Enrico Guerra ${ }^{4}$ Alessandro Marinelli ${ }^{4}$ Alessandra Menon ${ }^{1,2}$ \\ Pietro Simone Randelli ${ }^{1,2}$ Luigi Adriano Pederzini ${ }^{5}$ SIGASCOT Upper Limb Committee
}

\footnotetext{
${ }^{1}$ Dipartimento di Scienze Biomediche per la Salute, Università degli Studi di Milano, Milan, Italy

2 U.O.C. $1^{\text {a }}$ Divisione, Azienda Socio Sanitaria Territoriale Centro Specialistico Ortopedico Traumatologico Gaetano Pini-CTO, Milan, Italy,

${ }^{3}$ Department of Orthopaedics and Trauma Surgery, University of Bonn, Bonn, Germany

${ }^{4}$ Shoulder and Elbow Unit, Ortopedico Rizzoli, Bologna, Italy

${ }^{5}$ Arthroscopic and Sport Medical Center, Nuovo Ospedale di

Sassuolo, Sassuolo, Italy
}

Joints 2017;5:147-151.

\begin{abstract}
Address for correspondence Davide Cucchi, MD, Dipartimento di Scienze Biomediche per la Salute, Università degli Studi di Milano, Via Mangiagalli 31, Milan 20133, Italy (e-mail: d.cucchi@gmail.com).
\end{abstract}

\section{Abstract}

Keywords

- elbow arthroscopy

- posterior interosseous nerve

- nerve injury

- cadaver

Purpose The aims of this study were to measure the distance of the posterior interosseous nerve (PIN) from the radial head ( $\mathrm{RH}$ ) and its variations with forearm movements.

Methods Five fresh frozen cadaver specimens were dissected under arthroscopy. An anterior capsulectomy extended to the entire lateral compartment was performed. The need of soft tissue dissection to isolate the nerve in the extracapsular space was recorded. The distance between the nerve and the anterior part of the $\mathrm{RH}$ was then measured with a graduated caliper inserted via the midlateral portal with the forearm in neutral position, full pronation, and full supination.

Results The PIN was identifiable in all the specimens. In four cases, it was surrounded by a thick layer of adipose tissue, and further dissection was necessary to isolate it. Damage of the PIN during dissection occurred in one case, in which the proximal part of the nerve was accidentally cut. In three of the remaining cases, an increased distance was measured with the forearm in supination, as compared with neutral and full pronation position.

Conclusion This anatomical study suggests that in most of the cases, the PIN does not lay just extracapular at the level of the radiocapitellar joint, but is surrounded by a thick layer of adipose tissue. Furthermore, its distance from the RH appears to increase with forearm supination. This position could increase the safe working space between $\mathrm{RH}$ and PIN.

Clinical Relevance Knowledge of PIN position in relation to the anterior elbow capsule and its changes with forearm movements can help reduce the iatrogenic injuries during elbow arthroscopy.
\end{abstract}

${ }^{*}$ P. Arrigoni and D. Cucchi contributed equally to this work.

published online August 4, 2017
DOI https://doi.org/ $10.1055 / \mathrm{s}-0037-1605388$. ISSN 2282-4324.
Copyright @ 2017 Georg Thieme

Verlag KG Stuttgart · New York
License terms

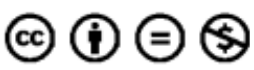




\section{Introduction}

The posterior interosseous nerve (PIN) innervates the supinator muscle and the extensor muscles of the wrist and the digits. A lesion to this nerve can occur after trauma and during open or arthroscopic elbow surgery. It may have dramatic consequences on forearm function and hence negatively affect the surgical outcome of procedures to the anterior and lateral aspect of the elbow. ${ }^{1-7}$

Although detailed measurements of PIN course have been provided from open surgical approaches, arthroscopic descriptions are lacking. ${ }^{8-11}$ Moreover, because of the different surgical position and setup used, conclusions derived from open surgery cannot be directly transferred into arthroscopic practice. For instance, the position of the elbow (90 degrees flexion and hanging with gravity when operating in prone or modified lateral decubitus) and the presence of intra-articular irrigation may influence the nerve displacement to a more anterior-inferior direction.

The primary aim of this study was to measure the distance of the PIN from the radial head ( $\mathrm{RH}$ ) during arthroscopic cadaver dissection and assess if PIN-RH distance varied with the forearm in a fully pronated and fully supinated position. The hypothesis of the study was that forearm movements could affect PIN-RH distance.

\section{Methods}

Five fresh frozen cadaver specimens were dissected under arthroscopy. Arthroscopy was performed with the elbow positioned at 90 degrees of flexion, with the hand and forearm hanging free with only gravity force. Standard posterior, posterolateral, and midlateral portals were first established to explore the posterior compartment, posteromedial gutter, and posterior aspect of the radiocapitellar joint. The anterior compartment of the elbow was then entered. A proximal anteromedial portal was created $2 \mathrm{~cm}$ proximal to the medial humeral epicondyle and $1 \mathrm{~cm}$ anterior to the intramuscular septum. Insertion of a 30-degree arthroscope into this portal allowed intra-articular diagnostic evaluation. An anterior capsulectomy extending into the lateral compartment was performed through a standard arthroscopic meniscal biter via the anteromedial approach while looking from the anterolateral portal. Particular attention was paid to identify the PIN immediately after anterior capsulectomy in the extracapsular space. If additional soft tissue dissection was necessary to isolate the radial nerve, the presence and quality of the interposed tissue was recorded.

After identification of the PIN, the distance between the nerve and the most anterior part of the radial head was measured with a graduated caliper inserted via the midlateral portal with the forearm in neutral position ( $\mathrm{PIN}-\mathrm{RH}_{\mathrm{n}}$ ) and expressed in centimeters (-Fig. 1). Subsequently, the forearm was moved first in full pronation and then in full supination; the same measurement was performed and recorded ( $\mathrm{PIN}-\mathrm{RH}_{\mathrm{p}}$ and $\mathrm{PIN}-\mathrm{RH}_{\mathrm{s}}$, respectively). Raw data were then normalized for the interepicondylar distance to account for anthropometric variations.

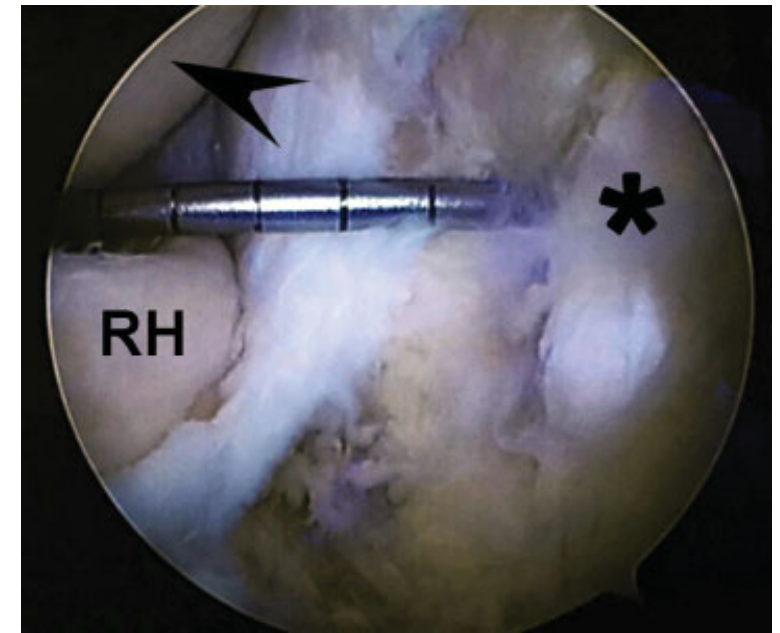

Fig. 1 Arthroscopic measurement of the distance between the anterior part of the $\mathrm{RH}$ and the posterior interosseous nerve (asterisk) with a graduated caliper inserted through the midlateral portal. Arrowhead indicates capitellum. RH; radial head.

All dissections were conducted by a group of surgeons experienced both in open and arthroscopic elbow procedures. Statistical analysis was performed using GraphPad Prism v 6.0 software (GraphPad Software Inc.). The Shapiro-Wilk normality test was used to evaluate the normal distribution of the sample; a nonparametric test (Friedman's test) was used to assess within-group differences and to compare measurements repeated in different conditions. Continuous variables were expressed as median and interquartile range (first and third quartiles) and or as mean \pm standard deviation as appropriate.

\section{Results}

Five specimens (median age: 83 years; range: 77-84; left elbow: 60\%) were evaluated. Mean interepicondylar distance was $7.26 \pm 1.78 \mathrm{~cm}$. The PIN was identifiable in all specimens. Damage of the PIN during dissection occurred in one case, in which the proximal part of the nerve was accidentally cut. In this case, the $\mathrm{PIN}-\mathrm{RH}_{\mathrm{p}}$ and $\mathrm{PIN}-\mathrm{RH}_{\mathrm{s}}$ distances could not be measured and were not included in further analysis. In all other cases, PIN-RH, $\mathrm{PIN}_{\mathrm{R}} \mathrm{RH}_{\mathrm{p}}$, and PIN-RH could be measured successfully.

In three cases, an increased distance in $\mathrm{PIN}^{-\mathrm{RH}_{\mathrm{s}}}$ as compared with $\mathrm{PIN}-\mathrm{RH}_{\mathrm{n}}$ and $\mathrm{PIN}-\mathrm{RH}_{\mathrm{p}}$ was noted, although no significant difference could be identified ( - Table 1 ).

Only in one specimen, the identification occurred immediately after capsulectomy. In the other four cases, it was necessary to isolate the nerve by further dissection. The tissue separating the nerve from the anterior elbow capsule was adipose in the four mentioned specimens (-Fig. 2).

\section{Discussion}

This anatomical study showed that the distance of the PIN from the RH can vary with forearm movements and suggested that supination could increase the PIN-RH distance, when compared with pronation and neutral position. Moreover, this 
Table 1 Absolute distance in centimeters of PIN-RH, normalized for interepicondylar distance with forearm in $\mathrm{PIN}_{\mathrm{R}} \mathrm{RH}_{\mathrm{n}}, \mathrm{PIN}_{\mathrm{R}} \mathrm{RH}_{\mathrm{p}}$, and PIN-RHs

\begin{tabular}{|l|l|l|l|}
\hline Specimen & ${\mathrm{PIN}-R H_{\mathbf{n}}}$ & ${\mathrm{PIN}-\mathrm{RH}_{\mathbf{p}}}$ & ${\mathrm{PIN}-\mathrm{RH}_{\mathbf{s}}}$ \\
\hline 1 & 0.23 & 0.15 & 0.30 \\
\hline 2 & 0.10 & 0.07 & 0.12 \\
\hline 3 & 0.38 & 0.54 & 0.31 \\
\hline 5 & 0.26 & 0.18 & 0.37 \\
\hline Mean \pm SD & $0.24 \pm 0.12$ & $0.23 \pm 0.21$ & $0.27 \pm 0.11$ \\
\hline $\begin{array}{l}\text { Median } \\
(\text { IQR) }\end{array}$ & 0.25 & 0.16 & 0.31 \\
$(0.19-0.29)$ & $(0.13-0.27)$ & $(0.26-0.32)$ \\
\hline
\end{tabular}

Abbreviations: IQR, interquartile range; PIN, posterior interosseous nerve; $\mathrm{RH}$, radial head; SD, standard deviation.

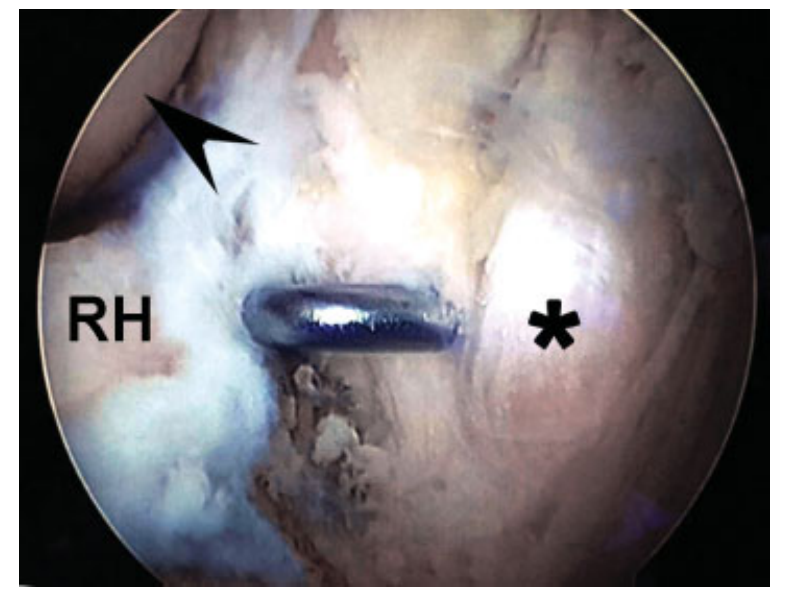

Fig. 2 The posterior interosseous nerve (asterisk) is separated from the anterolateral elbow capsule by a thick layer of adipose tissue, which is retracted by a probe inserted through the posterolateral portal. Arrowhead indicates capitellum. $\mathrm{RH}$, radial head.

study suggested that at the level of the radiocapitellar joint, the PIN did not lay just extracapsular, as previously described, but was surrounded by a layer of adipose tissue. Due to the small sample size, no significant difference could be identified in the study population, and therefore, larger studies with adequate sample size are required to confirm these conclusions.

The PIN is a motor nerve, which originates from the radial nerve after it separates from its superficial, sensory branch distally in the arm. ${ }^{10,12,13}$ In its proximal region, the PIN is separated from the joint capsule by the brachialis muscle; distally, when crossing the level of the $\mathrm{RH}$, no muscle bellies divide this structure from the joint, and only a thin layer of adipose tissue can be identified. Afterward, the PIN passes below the arcade of Frohse and continues its course toward the supinator muscle and the extensor muscles of the wrist and the digits, which are innervated by its fibers. ${ }^{10,14,15}$

In open surgery, the nerve is most vulnerable when passing below the arcade of Froshe and between the fibers of the supinator muscle. Nerve injury can be avoided by maintaining the forearm in pronation and approaching the
RH by either stripping the supinator from the radius (without exposing the PIN) or dividing its superficial humeral head to expose the PIN. ${ }^{16-19}$

Knowing the relative distance of the PIN to the RH and its variations with different forearm positions is a precious help in defining a safe working area in elbow arthroscopy. Previous studies on the variation of PIN position with forearm movements have only been performed in open surgery models with volar, dorsal, and lateral approaches. These studies demonstrated that a safe zone between the radiocapitellar joint to the point where the PIN crossed the lateral midline existed and its width decreased substantially with forearm movements from pronation to supination. ${ }^{8,13,15,20,21}$ No arthroscopic descriptions of the PIN position and its movements with respect to the most anterior part of the RH have been published yet.

The PIN is exposed to risk of injury during arthroscopy in two surgical steps: during portal establishment, since it passes close to the anterolateral portal, and during procedures on the $\mathrm{RH}$. While in its most proximal region, it is protected by the brachialis muscle, at the level of the RH, the PIN is separated from the joint capsule just by a layer of adipose tissue. ${ }^{13,22}$ Injury to the PIN is a known complication in elbow arthroscopy; ${ }^{6,23}$ however, a recent survey among the American Society for Surgery of the Hand members on major nerve injuries after elbow arthroscopy indicated this complication as under reported in the current literature. ${ }^{24}$ Our experience confirms that accidental lesion of the PIN may also happen easily to experienced surgeons. Therefore, we advise extreme care when performing elbow arthroscopy and suggest some tricks to reduce the risk of iatrogenic injury.

To prevent injuries in portal placement, we recommend to locate the anterolateral portal with an out-in technique, checking the needle entry point from the anteromedial portal, and to carefully incise the skin and subcutaneous tissues before inserting the arthroscope sheath and trocar or to locate this portal through an in-out technique. ${ }^{25,26}$ While establishing this portal, pronation displaces the nerve anteromedially, increasing safety on the chosen trajectory. ${ }^{21,27}$

To avoid accidental injury during intra-articular procedures, it is recommended to inject fluid to distend the capsule and displace the neurovascular structures away from the joint and orient the shaver or burr posteriorly. Inserting a retractor from a proximal anterolateral portal aimed at the radiocapitellar joint is another protective solution. ${ }^{28,29}$ Although no statistical significance could be obtained due to the small sample size, the results of this study suggested that supination could increase the distance of the PIN from the RH. Therefore, we agree in recommending pronation of the forearm whenever performing lateral extra-articular procedures (open approaches or portal placement), but recommend to supinate the forearm when working intraarticularly to increase the safe working space between the most anterior part of the RH and the PIN.

Future imaging studies with ultrasound or magnetic resonance imaging could help in clarifying the static and dynamic behavior of this nerve with respect to the $\mathrm{RH}$.

Open dissections indicate that while in its most proximal region, it is protected by the brachialis muscle, at the level of the $\mathrm{RH}$, the PIN lays in a very extracapsular space in front of 
the $\mathrm{RH}$, separated from the capsule just by a layer of adipose tissue. $8,9,13,16,20,22,30$

Assuming this statement, during standard arthroscopic surgery, precautions are taken considering the joint capsule to be the only protection from injuring the nerve in the space anterior to the RH. ${ }^{8-11}$ This study demonstrated that a thick layer of adipose tissue could be frequently encountered between the joint capsule and the PIN. This means that the safe zone between capsule and PIN is wider than expected from open cadaveric dissections, and therefore inadvertent shaving beyond the capsule layer can be forgiving in some cases. Nonetheless, we recommend using extreme care whenever working on the anterolateral aspect of elbow joint, taking all aforementioned precautions to avoid iatrogenic PIN injury.

Some authors recommended isolating the nerve in preparation for intra-articular procedures; in this study, in one specimen, the arthroscopic dissection, aimed at nerve identification, led to unexpected nerve damage. As a layer of adipose tissue protects this nerve from inadvertent excessive capsular shaving and nerve isolation may damage it, we advise against a routine dissection of the PIN, unless specifically indicated for the surgical procedure.

Limitations of this study include small sample size; this does not allow differentiating between any anatomical variants and may amplify bias related to technical aspects of the dissection. To reduce these, all the arthroscopies were performed by surgeons experienced in the field of elbow surgery. The nature of the study did not allow investigating on local pathologies of the capsule or systemic diseases that may influence soft tissue quality. The age of the specimens examined differs from the age range of patients undergoing elective arthroscopic elbow surgery, suggesting care when translating this anatomical data to a younger population. However, we suppose the behavior of this structure not to be directly affected by age depending tissue changes. Finally, we did not evaluate the effect of fluid extravasation on changes in the structural quality of the adipose tissue; in particular, we suppose that the length of the surgical procedure, especially with prolonged forceful positions, could influence adipose tissue distension and softness. ${ }^{31}$

In conclusion, PIN injuries are a serious complication of elbow surgery, and the knowledge of the working space is critical in avoiding them during arthroscopy. Although no statistical significance could be obtained due to the small sample size, this study suggests that the PIN assumes variable positions with respect to the $\mathrm{RH}$ when the forearm is moved, with a tendency toward increasing its distance in supination. Moreover, this study suggests that in most of the cases, the PIN does not lay just extracapular at the level of the radiocapitellar joint, but is surrounded by a thick layer of adipose tissue. Dissection aimed to isolate the nerve may create anyway further iatrogenic damage and is not indicated unless specifically needed for the surgical procedure.

\section{References}

1 Daurka J, Chen A, Akhtar K, Kamineni S. Tardy posterior interosseous nerve palsy associated with radial head fracture: a case report. Cases J 2009;2(01):22
2 Erra C, De Franco P, Granata G, et al. Secondary posterior interosseous nerve lesions associated with humeral fractures. Muscle Nerve 2016;53(03):375-378

3 Van Geertruyden JP, Vico PG. Iatrogenic posterior interosseous nerve palsy following an elbow fracture. Acta Orthop Belg 1996; 62(04):222-224

4 Saldua N, Carney J, Dewing C, Thompson M. The effect of drilling angle on posterior interosseous nerve safety during open and endoscopic anterior single-incision repair of the distal biceps tendon. Arthroscopy 2008;24(03):305-310

5 Lo EY, Li CS, Van den Bogaerde JM. The effect of drill trajectory on proximity to the posterior interosseous nerve during cortical button distal biceps repair. Arthroscopy 2011;27(08):1048-1054

6 Gupta A, Sunil Tm Tm. Complete division of the posterior interosseous nerve after elbow arthroscopy: a case report. J Shoulder Elbow Surg 2004;13(05):566-567

7 Mercer DM, Baldwin ED, Moneim MS. Posterior interosseous nerve laceration following elbow arthroscopy. J Hand Surg Am 2015;40(03):624-626

8 Diliberti T, Botte MJ, Abrams RA. Anatomical considerations regarding the posterior interosseous nerve during posterolateral approaches to the proximal part of the radius. J Bone Joint Surg Am 2000;82(06):809-813

9 Tornetta P III, Hochwald N, Bono C, Grossman M. Anatomy of the posterior interosseous nerve in relation to fixation of the radial head. Clin Orthop Relat Res 1997;(345):215-218

10 Ozkan M, Bacakoğlu AK, Gül O, Ekin A, Mağden O. Anatomic study of posterior interosseous nerve in the arcade of Frohse. J Shoulder Elbow Surg 1999;8(06):617-620

11 Mazurek MT, Shin AY. Upper extremity peripheral nerve anatomy: current concepts and applications. Clin Orthop Relat Res $2001 ;(383): 7-20$

12 Clavert P, Lutz JC, Adam P, Wolfram-Gabel R, Liverneaux P, Kahn JL. Frohse's arcade is not the exclusive compression site of the radial nerve in its tunnel. Orthop Traumatol Surg Res 2009;95(02): 114-118

13 Lawton JN, Cameron-Donaldson M, Blazar PE, Moore JR. Anatomic considerations regarding the posterior interosseous nerve at the elbow. J Shoulder Elbow Surg 2007;16(04):502-507

14 Thomas SJ, Yakin DE, Parry BR, Lubahn JD. The anatomical relationship between the posterior interosseous nerve and the supinator muscle. J Hand Surg Am 2000;25(05):936-941

15 Spinner M. The arcade of Frohse and its relationship to posterior interosseous nerve paralysis. J Bone Joint Surg Br 1968;50(04): 809-812

16 Kaplan E. Surgical approach to the proximal end of the radius and its use in fractures of the head and neck of the radius. J Bone Joint Surg 1941;23:86-92

17 Heidari N, Kraus T, Weinberg AM, Weiglein AH, Grechenig W. The risk injury to the posterior interosseous nerve in standard approaches to the proximal radius: a cadaver study. Surg Radiol Anat 2011;33(04):353-357

18 Strauch RJ, Rosenwasser MP, Glazer PA. Surgical exposure of the dorsal proximal third of the radius: how vulnerable is the posterior interosseous nerve? J Shoulder Elbow Surg 1996;5(05): 342-346

19 Schimizzi A, MacLennan A, Meier KM, Chia B, Catalano LW III, Glickel SZ. Defining a safe zone of dissection during the extensor digitorum communis splitting approach to the proximal radius and forearm: an anatomic study. J Hand Surg Am 2009;34(07): 1252-1255

20 Calfee RP, Wilson JM, Wong AH. Variations in the anatomic relations of the posterior interosseous nerve associated with proximal forearm trauma. J Bone Joint Surg Am 2011;93(01): $81-90$

21 Strachan JC, Ellis BW. Vulnerability of the posterior interosseous nerve during radial head resection. J Bone Joint Surg Br 1971; 53(02):320-323 
22 Kelly EW, Morrey BF, O'Driscoll SW. Complications of elbow arthroscopy. J Bone Joint Surg Am 2001;83-A(01):25-34

23 Carofino BC, Bishop AT, Spinner RJ, Shin AY. Nerve injuries resulting from arthroscopic treatment of lateral epicondylitis: report of 2 cases. J Hand Surg Am 2012;37(06):1208-1210

24 Desai MJ, Mithani SK, Lodha SJ, Richard MJ, Leversedge FJ, Ruch DS. Major peripheral nerve injuries after elbow arthroscopy. Arthroscopy 2016;32(06):999-1002.e8

25 Chaware PN, Santoshi JA, Pakhare AP, Rathinam BA. Risk of nerve injury during arthroscopy portal placement in the elbow joint: a cadaveric study. Indian J Orthop 2016;50(01):74-79

26 Blonna D, Wolf JM, Fitzsimmons JS, O’Driscoll SW. Prevention of nerve injury during arthroscopic capsulectomy of the elbow utilizing a safety-driven strategy. J Bone Joint Surg Am 2013; 95(15):1373-1381
27 Marshall PD, Fairclough JA, Johnson SR, Evans EJ. Avoiding nerve damage during elbow arthroscopy. J Bone Joint Surg Br 1993;75 (01):129-131

28 Pederzini LA, Nicoletta F, Tosi M, Prandini M, Tripoli E, Cossio A. Elbow arthroscopy in stiff elbow. Knee Surg Sports Traumatol Arthrosc 2014;22(02):467-473

29 Savoie FH III. Guidelines to becoming an expert elbow arthroscopist. Arthroscopy 2007;23(11):1237-1240

30 Jackson RW, Chow JCY. Advanced Arthroscopy. New York: Springer; 2012

31 Links AC, Graunke KS, Wahl C, Green JR III, Matsen FA III. Pronation can increase the pressure on the posterior interosseous nerve under the arcade of Frohse: a possible mechanism of palsy after twoincision repair for distal biceps rupture-clinical experience and a cadaveric investigation. J Shoulder Elbow Surg 2009;18(01):64-68 